\title{
OPINIONES
}

\section{Propuesta teórica de rehabilitación de bosques de Nothofagus pumilio degradados por incendios basada en plantaciones en núcleo con Embothrium coccineum}

\author{
Theoretical proposal for the rehabilitation of Nothofagus pumilio forests degraded \\ by fires based on cluster plantings with Embothrium coccineum
}

\author{
Alejandro Huertas-Herrera ${ }^{\mathrm{a}, \mathrm{b} *}$, Álvaro Promis ${ }^{\mathrm{c}}$, Matías Río ${ }^{\mathrm{d}}$, \\ Mónica Toro-Manríquez ${ }^{\text {a,b }}$, María Vanessa Lencinas ${ }^{\mathrm{e}}$, Guillermo Martínez Pastur ${ }^{\mathrm{e}}$ \\ *Autor de correspondencia: ${ }^{a}$ Centro de Investigación en Ecosistemas de la Patagonia (CIEP), \\ Camino Baguales s/n Km 4, Coyhaique, Chile, alejandro.huertas@ciep.cl \\ ${ }^{b}$ Ulterarius Consultores Ambientales y Científicos Ltda. Río de Los Ciervos 5862, \\ Loteo D, km 6 1⁄2 Sur, Punta Arenas, Chile. \\ ${ }^{c}$ Universidad de Chile, Facultad de Ciencias Forestales y de la Conservación de la Naturaleza, \\ Departamento de Silvicultura y Conservación de la Naturaleza, Santa Rosa 11315, Santiago, Chile. \\ d Fundación Reforestemos Patagonia. Del Inca 4622, Santiago, Chile. \\ ${ }^{\text {e }}$ Laboratorio de Recursos Agroforestales, Centro Austral de Investigaciones Científicas (CADIC), \\ Consejo Nacional de Investigaciones Científicas y Técnicas (CONICET), Houssay 200, Ushuaia (9410), \\ Tierra del Fuego, Argentina.
}

\section{SUMMARY}

The rehabilitation of temperate forests with native species requires trial and guidelines to achieve recovery of degraded areas of southern Chile and counteract the environmental imbalance caused by the impacts received. This study aims at presenting a theoretical proposal of rehabilitation based on cluster plantings to improve the recovery of lenga (Nothofagus pumilio) forests degraded by historical fires in Aysén Region. A rehabilitation strategy based on cluster plantings is proposed, in which individuals of lenga are established together with notro (Embothrium coccineum), the latter as a nurse plant, to accelerate the development of lenga seedlings through facilitation mechanisms, such as the improvement of microclimatic conditions, and protection from possible damage by seedling herbivores within each cluster. This proposal could be used in degraded lenga forests, both in Protected Wild Areas and in productive forests, as well as in landscapes fragmented by historical fires.

Key words: fire, protected wild areas, conservation, restoration, Patagonia.

\section{RESUMEN}

La rehabilitación de bosques templados con especies nativas requiere de ensayos y directrices que permitan alcanzar la recuperación de áreas degradadas del sur de Chile y contrarrestar el desequilibrio ambiental producto de los impactos recibidos. Este documento tiene como objetivo presentar una propuesta teórica de rehabilitación basada en plantaciones en núcleo para mejorar las potencialidades de recuperación de bosques de lenga (Nothofagus pumilio) degradados por incendios en la región de Aysén. La estrategia propone núcleos de lenga rodeados de notro (Embothrium coccineum), que actuaría como planta nodriza para acelerar el desarrollo de plántulas de lenga a través de mecanismos de facilitación, como el mejoramiento de las condiciones microclimáticas, y la protección de posibles daños por herbívoros de las plántulas dentro de cada núcleo. Esta propuesta podría ser utilizada en bosques degradados de lenga, tanto en Áreas Silvestres Protegidas como en bosques productivos, así como en paisajes fragmentados por incendios históricos.

Palabras clave: fuego, áreas silvestres protegidas, conservación, restauración, Patagonia.

\section{INTRODUCCIÓN}

Los bosques ocupan cerca del $31 \%$ del área terrestre a escala mundial (FAO 2020), desempeñando un papel pri- mordial en la mitigación del cambio climático, así como en el suministro de productos y servicios ecosistémicos esenciales para la humanidad (Acharya et al. 2019). Sin embargo, el continuo crecimiento de la población humana y el 
creciente consumo se han traducido en una explotación insostenible de la diversidad biológica, agravada por el cambio climático y otros impactos ambientales antropogénicos (Rands et al. 2010). En Chile, se han generado impactos ambientales considerables, con la consiguiente pérdida y fragmentación del hábitat, la invasión de especies exóticas y la explotación de los recursos naturales más allá de la resiliencia de los ecosistemas (Vidal et al. 2015, Miranda et al. 2017). Por ejemplo, entre 1936 y 1956, como respuesta al proceso de habilitación de tierras públicas para uso agrícola impulsado por el Estado de Chile, se quemaron alrededor de 3.500.000 hectáreas de bosques patagónicos en la región de Aysén (Quintanilla 2005). Cabe destacar que el fuego no forma parte de la dinámica natural de estos bosques, registrándose incendios solo como consecuencia de infrecuentes erupciones volcánicas o relámpagos, o por la llegada de las primeras poblaciones de humanos a la Patagonia (González et al. 2014, Mundo et al. 2017).

Los bosques patagónicos se caracterizan por la presencia de especies del género Nothofagus (Donoso 1993). Una de ellas es la lenga (Nothofagus pumilio [Poepp. et Endl.] Krasser), que se extiende desde los $35^{\circ} 35^{\prime} \mathrm{S}$ hasta los $56^{\circ} 00^{\prime} \mathrm{S}$ en la Región de Magallanes, incluyendo el archipiélago de Tierra del Fuego (González et al. 2006). La misma presenta un rango altitudinal desde el nivel del mar hasta los $2.000 \mathrm{~m} \mathrm{~s}$. n. m., el que tiende a disminuir desde el norte hacia el sur (Premoli 2004), y cuyo sotobosque se caracteriza por albergar pocas especies arbustivas, pero abundantes plantas herbáceas, helechos, musgos, hepáticas y hongos (Martínez Pastur et al. 2019). A pesar de su plasticidad genotípica y fenotípica que le permite distribuirse ampliamente en latitud y altitud (Premoli 2004), la lenga carece de estrategias regenerativas para hacer frente a los efectos adversos de los incendios, como la regeneración por rebrote (Veblen et al. 2003). Esto, sumado a otras condiciones desfavorables luego de los incendios en las regiones de Aysén y de Magallanes (Clewell 2015, Vidal et al. 2015), es una de las causas por las cuales la especie no ha logrado recuperarse de forma natural (González et al. 2006). Por ejemplo, los incendios alteran las propiedades físico-químicas del suelo (e.g. pH, nitrógeno total, fósforo disponible, materia orgánica) (Urretavizcaya et al. 2018), lo que repercute en pérdida de nutrientes, y disminución de la materia orgánica y de los ensambles biológicos que afectan el restablecimiento natural de lenga (Jaksic y Fariña 2015, Urretavizcaya et al. 2015).

Existen diversas propuestas para la recuperación de los bosques degradados donde no ocurre el proceso de regeneración natural. Para bosques de lenga se ha propuesto que en los sectores más expuestos y sin cobertura arbórea, como los que se pueden encontrar en bosques degradados por incendios, la supervivencia de la especie es mayor cuando se planta en grupos (Fajardo y McIntire 2011). Tales agrupaciones pueden estar relacionadas con una mejor adquisición de los recursos o una mejor estabilidad estructural contra el viento (Till-Bottraud et al. 2012). Así lo demuestran estudios en claros de dosel y en bosques manejados de lenga donde, por un lado, una mayor luminosidad en el claro estimula el proceso de regeneración y el crecimiento de individuos juveniles en bosques naturales (Fajardo y de Graaf 2004, Promis 2018) y, por otro lado, donde una mayor retención de árboles proporciona mejores condiciones (e.g. humedad de suelo) para una mayor densidad de nuevos individuos (Martínez Pastur et al. 2019). Así, los núcleos funcionan como un mecanismo de facilitación de las plantas (Fajardo y McIntire 2011).

Por otro lado, se reconoce ampliamente que las plantas nodrizas facilitan el establecimiento y crecimiento de numerosas especies (Holmgren y Scheffer 2010, Delgado et al. 2015). El uso de arbustos y árboles nodriza como facilitadores para la restauración de bosques de lenga, ha sido probado por Valenzuela et al. (2016). En condiciones ambientales de mayor estrés, se ha planteado que la facilitación tendría un efecto positivo (Holmgren y Scheffer 2010), a través de la generación de sombra (e.g. reducción de las tasas de evaporación en el suelo), aumento de la humedad ambiental, reducción del viento y de la temperatura del suelo (Whisenant 1999), mejora en la disponibilidad de nutrientes por fijación de nitrógeno y mayor aporte de hojarasca (Maestre et al. 2003). En este contexto, notro (Embothrium coccineum J. R. Forst. y G. Forst) podría ser una especie candidata para acompañar los núcleos de lenga, por ser una especie colonizadora temprana en la sucesión de vegetación (Escobar et al. 2006). Según Mathiasen et al. (2007) y Delgado et al. (2015), el notro tiene raíces proteoides que le permiten aumentar la superficie de absorción, obteniendo una mayor cantidad de agua y nutrientes del suelo, principalmente fósforo. Esto se consigue secretando ácidos y enzimas que solubilizan dicho nutriente del sustrato inorgánico (Piper et al. 2013). Dicha conversión ocurre en la rizósfera, por lo que esta especie posiblemente permita el aprovechamiento de los nutrientes solubilizados por otras especies que crezcan a su alrededor (Rovere 2010, Delgado et al. 2015).

Dados los grandes esfuerzos que se ponen en los proyectos de rehabilitación y/o restauración (Valenzuela et al. 2015, Bannister et al. 2018), las expectativas y las cantidades de recursos que se invierten (Vidal et al. 2015), es preciso proponer y discutir nuevas ideas para mejorar la recuperación y conservación de los bosques templados degradados en el sur del país (Clewell 2015), como los de lenga o similares. El objetivo de este trabajo es presentar una propuesta teórica de rehabilitación basada en plantaciones en núcleo para la recuperación de bosques degradados por incendios de lenga en la región de Aysén.

\section{EJEMPLO PARA UNA RESERVA NACIONAL EN LA REGIÓN DE AYSÉN}

Modelo conceptual para el diagnóstico del estado de degradación. De acuerdo con los principios de recuperación de Whisenant (1999), es posible definir el estado de de- 
gradación de un bosque a rehabilitar en función del estado de la vegetación actual y su historia. Por ejemplo, en la región de Aysén (e.g. en la Reserva Nacional Lago Carlota, ubicada entre $44^{\circ} 27^{\prime}$ 'y $44^{\circ} 36^{\prime}$ S y entre $71^{\circ} 25^{\prime}$ ' $\mathrm{y} 71^{\circ} 44^{\prime}$ O), el Bosque Caducifolio caracterizado por el predominio de la lenga (Gajardo 1994), evidencia historia de incendios (figura 1A) con presencia de plantas nativas y/o exóticas típicas de sitios degradados por fuego, como Baccharis magellanica y Rumex acetosella (Silva 2010). Este tipo de situaciones presentaría un estado de degradación nivel 3 (figura 2), donde se han superado dos umbrales importantes de degradación: (1) un umbral controlado por factores bióticos, como la presencia de especies vegetales típicas de matorral o pradera que no deben encontrarse en bosques
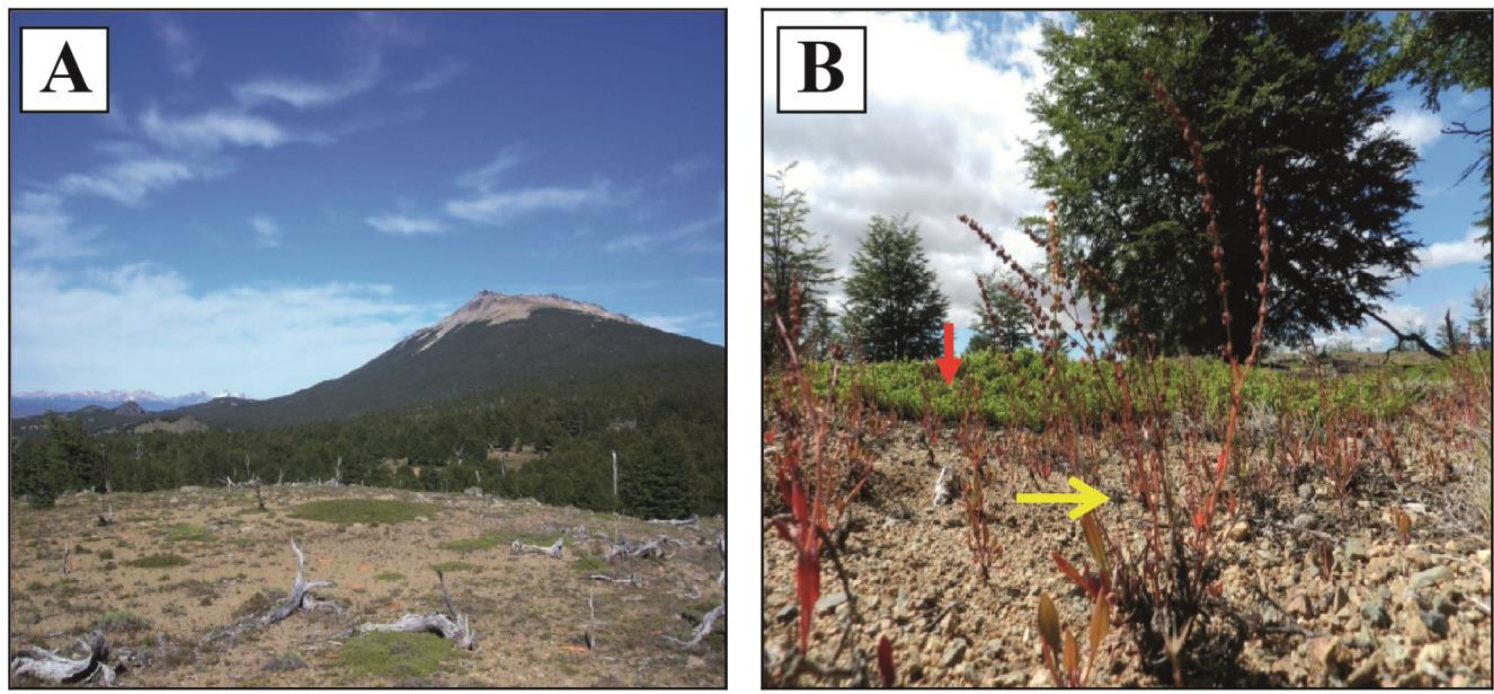

Figura 1. (A) Ejemplo de ambiente degradado por incendio en la Reserva Nacional Lago Carlota (región de Aysén). (B) Especies indicadoras de degradación por fuego (Silva 2010): Baccharis magellanica (flecha roja) y Rumex acetosella (flecha amarilla).

(A) Example of an environment degraded by fire in Lago Carlota National Reserve (Aysén Region). (B) Indicator species of degradation by fire (Silva 2010): Baccharis magellanica (red arrow) and Rumex acetosella (yellow arrow).

Umbral controlado

por factores bióticos

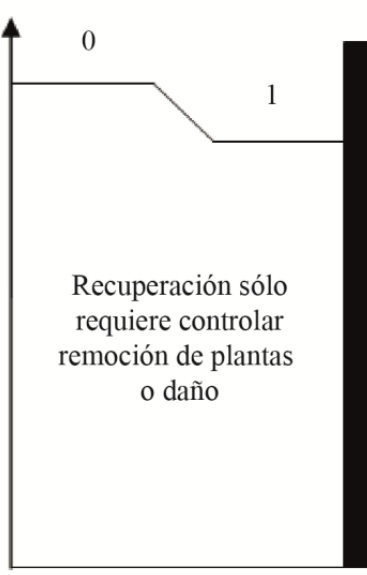

Umbral controlado

por factores abióticos

\section{Alta $\longleftarrow \begin{aligned} & \text { Eficiencia uso de agua } \\ & \text { Eficiencia captura de energía } \\ & \text { Eficiencia uso nutrientes }\end{aligned}$}

Procesos

primarios

funcionales

3 requiere
manipulación manipulación vegetación

Procesos

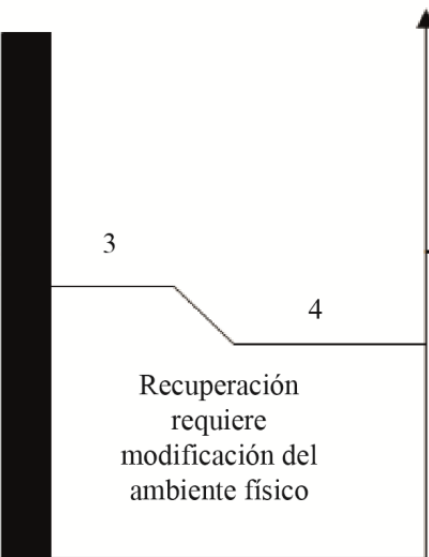

primarios no funcionales

Figura 2. Modelo teórico de degradación de ecosistemas naturales, donde los números indican los niveles de degradación (adaptado de Whisenant 1999).

Theoretical model of the degradation of natural ecosystems, where the numbers indicate the levels of degradation (adapted from Whisenant 1999). 
de lenga, y (2) un umbral controlado por factores abióticos, como la erosión eólica. Por ejemplo, en la figura 1 (A, B), se evidencia una situación en la que predomina suelo sin vegetación y/o con presencia de algunas especies como $B$. magellanica. Estas limitaciones físicas y bióticas implican la alteración de procesos fundamentales como: (1) el ciclo de nutrientes, (2) el flujo de agua y (3) la captura de energía o producción primaria neta. Para recuperar el bosque de este nivel de degradación es imperativo corregir los umbrales controlados por factores abióticos y bióticos, siendo necesarias varias modificaciones del ambiente como: (i) control de la erosión, (ii) protección de la superficie del suelo, (iii) mejora de las condiciones microambientales para el establecimiento de la regeneración, (iv) aumento de la capacidad de retención de agua y nutrientes, y (v) control estricto de la herbivoría.

\section{PROPUESTA TEÓRICA PARA LA REHABILITACIÓN DEL BOSQUE DEGRADADO}

De acuerdo con Corbin y Holl (2012), esta propuesta teórica de rehabilitación está basada en plantaciones en núcleos (figura 3A), que implica la plantación de dos espe- cies arbóreas nativas juntas (lenga y notro) en cada núcleo o grupo. Se recomienda que las plántulas, tanto de lenga como de notro, tengan $\sim 5 \mathrm{~mm}$ de diámetro a la altura del cuello de la raíz, 15 a $20 \mathrm{~cm}$ de altura, calidades similares (e.g. plántulas con un solo tallo), y proporción de masa raíz/tallo no menor a 2:1. Con base en el modelo de Saha et al. (2016), se propone plantar notro en el perímetro del núcleo (primeras filas), y en la zona central, las plántulas de lenga (figura 3B, C). Con el tiempo, se espera que la cobertura arbórea se expanda a través del crecimiento de los árboles y nuevas colonizaciones que ocurran espontáneamente (Corbin y Holl 2012), recuperando lentamente las áreas desprovistas de vegetación entre los núcleos a través de un proceso de sucesión.

Considerando a Schmidt et al. (2003) y Escobar et al. (2006), la figura 4 simula el desarrollo de etapas que se espera que sigan las plantaciones de lenga junto con el notro. En esta proyección no se recrean todas las especies vegetales originalmente presentes, sino aquellas que actuarán en la recuperación del medio. En este orden de ideas, para rehabilitar un área de 100 ha, se generarían 50 a 55 núcleos por hectárea, formando una red de núcleos separados $7 \mathrm{~m}$. Cada núcleo contendría 8 plántulas de lenga en el centro

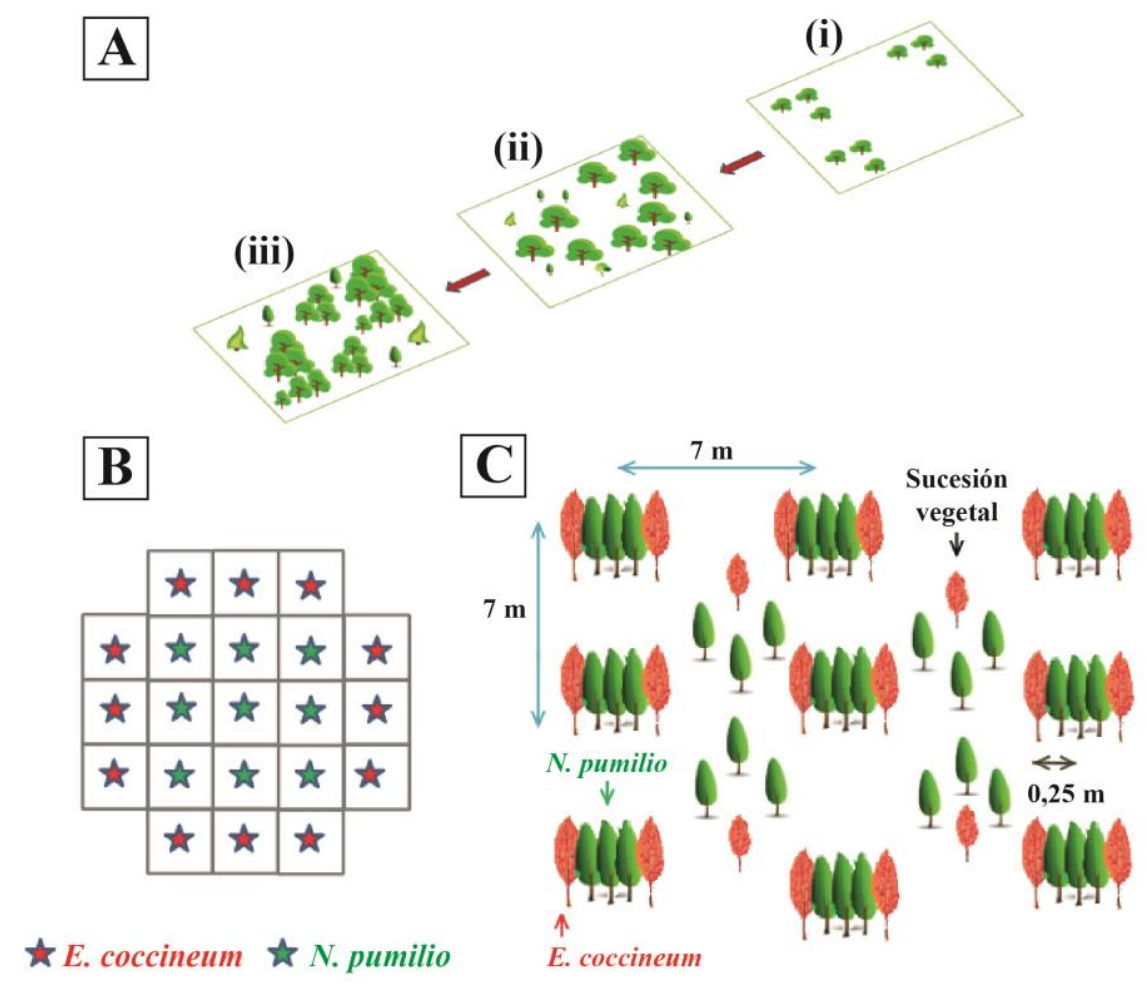

Figura 3. (A) Desarrollo de núcleos de rehabilitación en áreas degradadas (adaptado de Corbin y Holl 2012). En la etapa inicial se establecen los núcleos (i), que favorece el crecimiento de la regeneración (ii), que posteriormente conforman parches (iii). (B) Diseño para la distribución espacial de núcleos, con $7 \times 7 \mathrm{~m}$ entre ellos, y $(\mathrm{C})$ ejemplo de núcleo de plántulas plantadas de manera agregada (adaptado de Saha et al. 2016).

(A) Development of rehabilitation clusters in degraded areas (adapted from Corbin and Holl 2012). In the initial stage, the clusters are established (i), that favors the growth of regeneration (ii), which later form a unit patch (iii). (B) Design for the spatial distribution of clusters, with $7 \times 7$ $\mathrm{m}$ between them, and (C) example of a cluster planting (adapted from Saha et al. 2016). 
del núcleo y 12 plantas de notro en el borde. Cada planta dispondría de una superficie de $1 \mathrm{~m}^{2}$, teniendo el núcleo una superficie total de $20 \mathrm{~m}^{2}$. El propósito del núcleo es asegurar que al menos una planta de lenga alcance dimensiones importantes dentro del área de $20 \mathrm{~m}^{2}$. Así, para la fase de crecimiento óptima, se espera una densidad de al menos 50 árboles por hectárea. Para el control de herbívoros (e.g. liebres como Lepus europaeus Pallas, 1778), se propone la protección con tubos de protección (shelters). El uso de estos tubos de protección ha mostrado resultados positivos para el control de la herbivoría, permitiendo además mejorar las condiciones microambientales alrededor de la planta mediante la reducción de la fuerza del viento y el aumento de la humedad relativa en su interior (Oliet y Jacobs 2007, Oliet et al. 2019). Los tubos deberían ser retirados una vez las plantas alcancen una altura tal que le permita escapar del ramoneo $(\sim 1 \mathrm{~m})$, para evitar daños mecánicos y/o deformaciones de las plantas en etapas avanzadas de su crecimiento (Urretavizcaya et al. 2015). Otras actividades que podrían acompañar a esta propuesta se presentan en el cuadro 1 , con requerimientos variables de aportes pecuniarios y mano de obra, cuya evaluación supera los alcances de esta propuesta.

\section{CONSIDERACIONES Y RECOMENDACIONES}

Algunos autores sugieren que las plantaciones en grupo son propuestas útiles en sistemas en los que el establecimiento y desarrollo de las plántulas está limitado por condiciones de estrés ambiental (Corbin y Holl 2012). Para Shönenberger (2001), las plantaciones en grupo pueden llegar a tener varias ventajas, entre las cuales se menciona que: (i) la mortalidad de las plántulas debería ser más baja que en plantaciones tradicionales, con plántulas homogé-

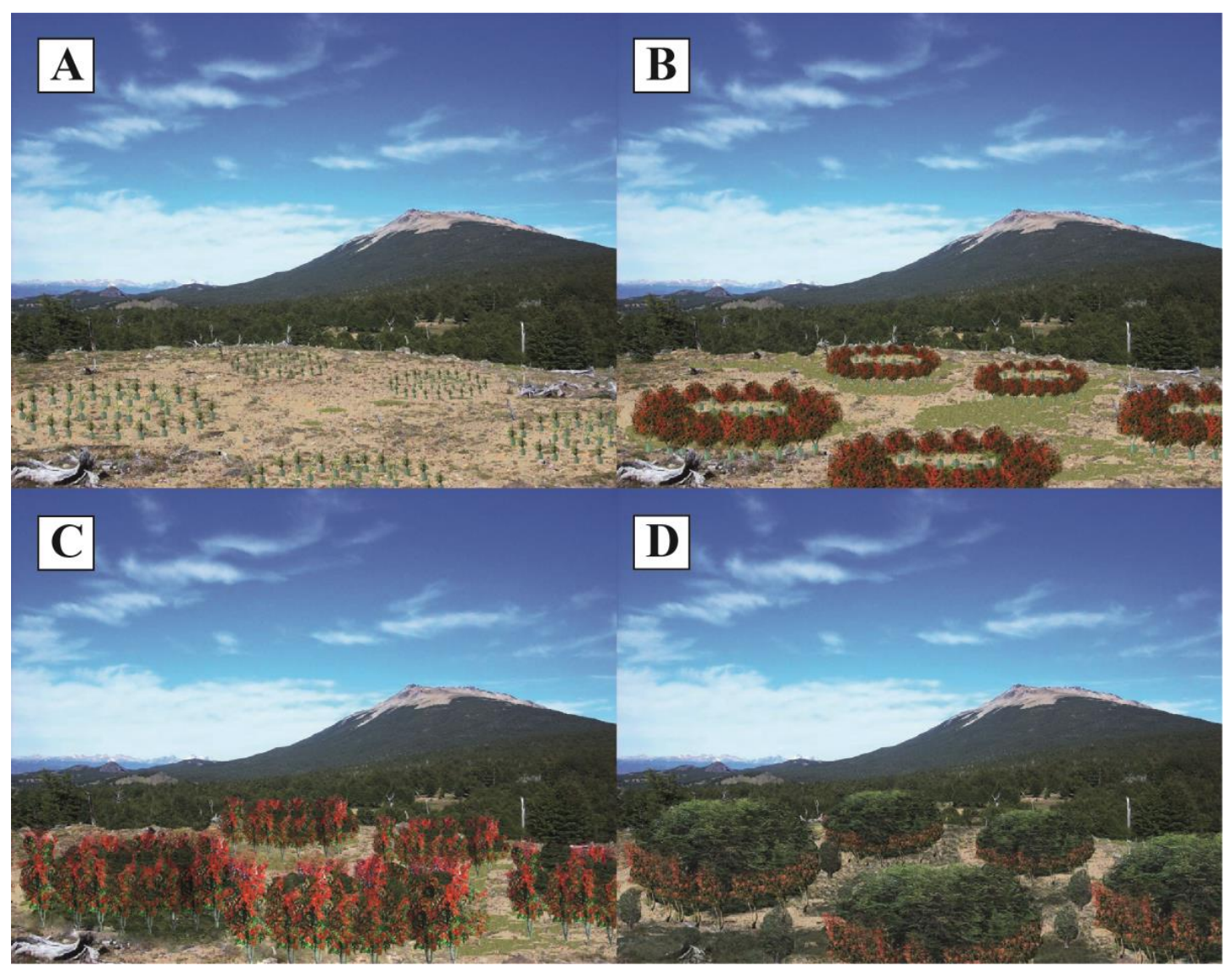

Figura 4. Simulación visual de las etapas de desarrollo esperadas con el establecimiento de núcleos: (A) establecimiento de la plantación donde se protegen las plantas periféricas (notro) y del centro (lenga) con tubos protectores; (B) plantación en 5 años, notro con 3 a 4 m, mientras que lenga alcanza $1 \mathrm{~m}$ de altura; (C) plantación en 25 años donde notro alcanza 6 a 7 m, mientras que lenga alcanza $4 \mathrm{~m}$ de altura; (D) plantación en 50 años donde notro alcanza $8 \mathrm{~m}$, mientras lenga alcanza entre 7 a $8 \mathrm{~m}$ de altura, y ya se espera regeneración natural de ambas especies e iniciación de abundante sotobosque.

Visual simulation of the stages of development expected with the establishment of cluster planting: (A) establishment of the cluster planting where peripheral (notro) and inner (lenga) individuals are protected with shelters; (B) plantation in 5 years, the notro reaches between 3 to $4 \mathrm{~m}$ in height, while the lenga reaches $1 \mathrm{~m}$ in height; (C) plantation in 25 years, the notro reaches 6 to $7 \mathrm{~m}$ high, while the lenga reaches $4 \mathrm{~m}$ high; (D) plantation in 50 years, the notro reaches $8 \mathrm{~m}$ in height, while the lenga reaches between 7 to $8 \mathrm{~m}$ in height, natural regeneration of both species and the initiation of understory is expected. 
Cuadro 1. Actividades que podrían acompañar a la propuesta de plantación en núcleos para la rehabilitación de áreas degradadas por incendios.

Activities that could accompany the proposed planting in clusters for the rehabilitation of areas degraded by fires.

\begin{tabular}{|c|c|c|}
\hline Actividad & Descripción y foco de la actividad & Literatura ejemplo \\
\hline Control Biológico & $\begin{array}{l}\text { Aumentar la densidad de aves rapaces, como el Bubo magellanicus, que incluye } \\
\text { en su dieta presas de gran tamaño como la liebre. Sería necesario atraer las } \\
\text { aves por medio de la construcción de cajas para nidos y perchas-posadera, } \\
\text { específicamente en los ambientes de pradera o lugares abiertos. }\end{array}$ & $\begin{array}{l}\text { Muñoz-Pedreros et } \\
\text { al. (1996). Celis-Diez } \\
\text { et al. (2011). }\end{array}$ \\
\hline Mejoramiento de suelos & $\begin{array}{l}\text { Mejorar la rugosidad del terreno empleando surcos de contorno en zonas de } \\
\text { pendiente y depresiones en las zonas planas. Se busca crear micrositios seguros } \\
\text { para las semillas de lenga. Además, utilizar troncos de los árboles muertos de } \\
\text { forma ordenada para capturar y retener nutrientes, favoreciendo la formación } \\
\text { de materia orgánica y retención de humedad necesaria para la germinación de } \\
\text { semillas. }\end{array}$ & $\begin{array}{l}\text { Valenzuela et al. } \\
(2015,2016) \text {. } \\
\text { Urretavizcaya et al. } \\
(2018) \text {. }\end{array}$ \\
\hline $\begin{array}{l}\text { Manejo de la vegetación } \\
\text { actual }\end{array}$ & $\begin{array}{l}\text { En las situaciones en que no ocurran invasiones de plantas problemáticas, se } \\
\text { propone no remover las plantas nativas e introducidas y mantener el efecto } \\
\text { pasivo de éstas en el área de la plantación de lenga, como la reducción de la } \\
\text { fuerza del viento y condiciones de micrositio. }\end{array}$ & $\begin{array}{l}\text { Valenzuela et } \\
\text { al. (2016). Toro } \\
\text { Manríquez et al. } \\
\text { (2018). }\end{array}$ \\
\hline Riego & $\begin{array}{l}\text { En lo posible, mejorar las condiciones hídricas de la plantación aplicando riego } \\
\text { con mangueras a las plántulas. }\end{array}$ & $\begin{array}{l}\text { Urretavizcaya et al. } \\
(2015) \text {. }\end{array}$ \\
\hline Cortinas cortavientos & $\begin{array}{l}\text { Establecer cortinas forestales en forma de cortavientos, donde los arbustos } \\
\text { nativos y notro se presentan como una alternativa en los sectores abiertos sin } \\
\text { bosque. }\end{array}$ & Peri (1998). \\
\hline $\begin{array}{l}\text { Participación de la } \\
\text { comunidad circundante }\end{array}$ & $\begin{array}{l}\text { Desarrollar actividades turísticas, promoviendo los atractivos naturales y } \\
\text { culturales, orientándose a un desarrollo turístico rural a través del trabajo } \\
\text { mancomunado con la municipalidad y los servicios turísticos ya existentes. }\end{array}$ & $\begin{array}{l}\text { Whisenant (1999). } \\
\text { Bannister et al. } \\
\text { (2018). }\end{array}$ \\
\hline
\end{tabular}

neamente distribuidas, y que pueden llegar a establecerse los grupos de plantaciones en micrositios con condiciones favorables para las plántulas; (ii) los crecimientos de las plántulas pueden llegar a ser mejores, debido a las condiciones microclimáticas que se pueden llegar a generar en cada uno de los grupos; (iii) las plántulas que están hacia fuera del grupo, tenderán a proteger a las plántulas centrales del posible daño de herbivoría; y (iv) las plántulas se protegerían de posibles daños por el viento y desecamiento invernal. De acuerdo con Saha et al. (2013), la técnica de plantación en núcleos complementada con la regeneración natural se consolida como un modo de reforestación que asegura el crecimiento de un número suficiente de árboles de la especie deseada, en la que se mejoran las condiciones circundantes al núcleo, permitiendo la regeneración de individuos que probablemente morirían por malas condiciones de sitio.

Debe tenerse en cuenta que la lenga crece mejor en suelos ricos en fósforo (Toro Manríquez et al. 2019), de manera que la falta de materia orgánica y nutrientes puede ser uno de los factores limitantes para la regeneración natural (Veblen et al. 2003, Promis y Allen 2017). Para superar este tipo de limitaciones, Whisenant (1999) ha propuesto orientar los procesos en torno a la sucesión natural, siendo importante crear o incrementar micrositios seguros en lugares disturbados, que son aquellos lugares donde las semillas pueden germinar en un conjunto de condiciones ambientales adecuadas para su posterior establecimiento y crecimiento (Toro Manríquez et al. 2018). Si bien no se cuenta con antecedentes que indiquen una positiva interacción entre la lenga y el notro, el proceso de facilitación aumenta bajo condiciones de estrés abiótico (Maestre et al. 2003), mientras que la competencia es más importante en ambientes con mayor oferta de recursos (Callaway 1997). Se sabe que el notro es una especie pionera en terrenos abiertos (Quintanilla 2005, Escobar et al. 2006), se asocia con lenga (Escobar et al. 2006) y es considerada como una especie facilitadora (Rovere 2010, Delgado et al. 2015). Esto concuerda con Piper et al. (2013), quienes han comprobado que las raíces de notro son capaces de capturar el fósforo, e incluso el nitrógeno en ambientes degradados (e.g. como los impactados por incendios y sobrepastoreo en la región de Aysén).

Finalmente, la creación y/o retención de elementos estructurales de los bosques, se ha propuesto como camino para acercar la producción a la conservación (Martínez Pastur et al. 2019). En este contexto, crear elementos a escala de rodal o retenerlos (recuperación de legados en 
el bosque degradado) mejoraría el nivel de conservación, principalmente en aquellos lugares (e.g. áreas protegidas) que apuntan a funciones ecosistémicas y de conservación de la biodiversidad. Estudios en bosques de lenga sugieren que determinados atributos del bosque manejado cumplen un rol importante para la conservación de la biodiversidad (e.g. Martínez Pastur et al. 2019). En concordancia, resultaría imperativo considerar como estrategias complementarias de rehabilitación de bosques de lenga los siguientes aspectos: (i) mantener en el sitio residuos que dan estructura al ecosistema (e.g. tocones, raíces, troncos en descomposición); (ii) retener y/o crear otros múltiples elementos estructurales (e.g. árboles en desmoronamiento que proporcionan hábitats únicos para la biodiversidad). Con base en Whisenant (1999), se propone remover las plantas introducidas problemáticas (invasoras) y mantener aquellas que puedan contribuir con la mejora del ecosistema (e.g. reducción de la fuerza del viento y condiciones de micrositio); (iii) establecer clausuras (mallas, cercos) en las que se proteja la regeneración de individuos, pastizales, y otros elementos del sotobosque, favoreciendo además la heterogeneidad de microambientes; (iv) mantener núcleos de vegetación (individuos vivos remanentes) (Martínez Pastur et al. 2013) y (v) crear pilas con restos leñosos que cumplan una función ecológica (e.g. percha de aves; microclima especies) (Whisenant 1999). Así pues, retener y/o crear elementos del bosque mejoraría la conservación $\mathrm{y}$ en el resto de las variables abióticas forestales a microescala, pues una distribución agrupada puede ofrecer una mayor estabilidad ecológica (Lindenmayer et al. 2015). Esta propuesta de rehabilitación se origina en el conocimiento profundo de una problemática local (bosques de lenga degradados por incendios en la región de Aysén) y de la respuesta ecológica de dos especies bastante estudiadas (lenga y notro). Sin embargo, creemos que es replicable a la rehabilitación de otros bosques degradados y otras especies, aun cuando fueran ecológicamente menos conocidas. Además, implica un diseño novedoso, que podría promover exitosos resultados para la restauración, como es deseable y necesario en muchos bosques en Chile (Bannister et al. 2018).

\section{AGRADECIMIENTOS}

Los autores agradecemos al equipo de Patagonia Sur (SNP) y al Grupo de Investigación Aplicada en Ecología y Conservación de la Vegetación (ECOVEG) de la Universidad de Chile, por el apoyo en el desarrollo de esta propuesta.

\section{REFERENCIAS}

Acharya RP, T Maraseni, G Cockfield. 2019. Global trend of forest ecosystem services valuation - An analysis of publications. Ecosystem Services 39: 100979. DOI: https://doi. org/10.1016/j.ecoser.2019.100979.
Bannister JR, R Vargas-Gaete, JF Ovalle, M Acevedo, A FuentesRamírez, PJ Donoso, A Promis, C Smith-Ramírez. 2018. Major bottlenecks for the restoration of natural forests in Chile. Restoration Ecology 26(6): 1039-1044. DOI: https:// doi.org/10.1111/rec.12880.

Callaway RM. 1997. Positive interactions in plant communities and the individualistic continuum concept. Oecologia 112: 143-149. DOI: https://doi.org/10.1007/s004420050293.

Celis-Diez JL, S Ippi, A Charrier, C Garrín. 2011. Fauna de los bosques templados de Chile. Guía de campo de los vertebrados terrestres. Corporación Chilena de la Madera. Concepción, Chile. 261 p.

Clewell AF. 2015. Ecological restoration principles relative to Nothofagus pumilio (Poepp. \& Endl.) Krasser (Nothofagaceae) forest restoration. Anales Instituto Patagonia 43(1): 123-126. http://dx.doi.org/10.4067/S0718686X2015000100010.

Corbin JD, KD Holl. 2012. Applied nucleation as a forest restoration strategy. Forest Ecology and Management 265: 37-46. DOI: https://doi.org/10.1016/j.foreco.2011.10.013.

Delgado M, A Zúñiga-Feest, F Borie. 2015. Ecophysiological role of Embothrium coccineum, a Proteaceae species bearing cluster roots, at increasing Phosphorus availability in its rhizosphere. Journal of Soil Science and Plant Nutrition 15(2): 307-320. DOI: https://doi.org/10.4067/S071895162015005000028.

Donoso C. 1993. Bosques templados de Chile y Argentina. Variación, estructura y dinámica. Editorial Universitaria. Santiago, Chile. 483 p.

Escobar B, C Donoso, C Souto, M Alberdi, A Zuñiga. 2006. Embothrium coccineum J.R. et. G. Forster Notro, Notru, Ciruelillo, Treumén, Fosforito. Familia: Preoteaceae. In Donoso $\mathrm{C}$ ed. Las especies arbóreas de los bosques templados de Argentina y Chile, autoecología. Valdivia, Chile. Marisa Cuneo Ediciones. p. 233-245.

FAO (Food and Agriculture Organization, Organización de las Naciones Unidas). 2020. Global Forest Resources Assessment 2020 - Key findings. Rome, Italia. FAO. 16 p. DOI: https://doi.org/10.4060/ca8753en.

Fajardo A, R de Graaf. 2004. Tree dynamics in canopy gaps in old-growth forests of Nothofagus pumilio in Southern Chile. Plant Ecology 173: 95-105. DOI: https://doi. org/10.1023/B:VEGE.0000026333.54741.97.

Fajardo A, EJB Mcintire. 2011. Under strong niche overlap conspecifics do not complete but help each other to survive: facilitation at the intraspecific level. Journal of Ecology 99(2): 642-650. DOI: https://doi.org/10.1111/j.13652745.2010.01771.x.

Gajardo R. 1994. La vegetación Natural de Chile: Clasificación y distribución geográfica, Santiago, Chile. Editorial Universitaria. $165 \mathrm{p}$.

González M, C Donoso, P Ovalle, G Martínez-Pastur. 2006. Nothofagus pumilio. In Donoso C ed. Las especies arbóreas de los bosques templados de Chile y Argentina: Autoecología. Valdivia, Chile. Marisa Cuneo Ediciones. p. 486-500.

González M, M Amoroso, A Lara, TT Veblen, C Donoso, T Kitzberger, I Mundo, A Holz, A Casteller, J Paritsis, A Muñoz, ML Suárez, A Promis. 2014. Ecología de disturbios y su influencia en los bosques templados de Chile y Argentina. In C Donoso, ME González, A Lara A eds. Ecología Forestal. Bases para el manejo sustentable y conservación de los bos- 
ques nativos de Chile. Valdivia, Chile. Ediciones UACh. p. 411-502.

Holmgren M, M Scheffer. 2010. Strong facilitation in mild environments: the stress gradient hypothesis revisited. Journal of Ecology 98(6): 1269-1275. DOI: https://doi.org/10.1111/ j.1365-2745.2010.01709.x.

Jaksic FM, JM Fariña. 2015. Incendios, sucesión y restauración ecológica en contexto. Anales Instituto Patagonia 43(1): 23-34. DOI: http://dx.doi.org/10.4067/S0718686X2015000100003.

Lindenmayer DB, J Wood, L McBurney, D Blair, SC Banks. 2015. Single large versus several small: The SLOSS debate in the context of bird responses to a variable retention logging experiment. Forest Ecology and Management 339: 1-10. DOI: https://doi.org/10.1016/j.foreco.2014.11.027.

Maestre FT, S Bautista, J Cortina. 2003. Positive, negative, and net effects in grass-shrub interactions in mediterranean semiarid grasslands. Ecology 84(12): 3186-3197. DOI: https://doi.org/10.1890/02-0635.

Martínez Pastur G, PL Peri, MV Lencinas, JM Cellini, M Barrera, R Soler, H Ivancich, L Mestre, AS Moretto, CB Anderson, F Pulido. 2013. La producción forestal y la conservación de la biodiversidad en los bosques de Nothofagus en Tierra del Fuego y Patagonia Sur. In Donoso P, Á Promis eds. Silvicultura en bosques nativos: Avances en la investigación en Chile, Argentina y Nueva Zelanda. Valdivia, Chile. Marisa Cuneo Ediciones. p. 155-179.

Martínez Pastur G, YM Rosas, M Toro Manríquez, A Huertas Herrera, JA Miller, JM Cellini, MD Barrera, PL Peri, MV Lencinas. 2019. Knowledge arising from long-term research of variable retention harvesting in Tierra del Fuego: where do we go from here? Ecological Processes 8(1): 24. DOI: https://doi.org/10.1186/s13717-019-0177-5.

Mathiasen P, AE Rovere, AC Premoli. 2007. Genetic structure and early effects of inbreeding in fragmented temperate forests of a self-incompatible tree, Embothrium coccineum. Conservation Biology 21(1): 232-240. DOI: https://doi. org/10.1111/j.1523-1739.2006.00565.x.

Miranda A, A Altamirano, L Cayuela, A Lara, M González. 2017. Native forest loss in the Chilean biodiversity hotspot: revealing the evidence. Regional Environmental Change 17: 285-297. DOI: https://doi.org/10.1007/s10113-016-1010-7.

Mundo IA, R Villalba, TT Veblen, T Kitzberger, A Holz, J Paritsis, A Ripalta. 2017. Fire history in southern Patagonia: human and climate influences on fire activity in Nothofagus pumilio forests. Ecosphere 8: e01932. DOI: https://doi. org/10.1002/ecs2.1932.

Muñoz-Pedreros A, A Gantz, M Saavedra. 1996. Nidos artificiales en plantaciones de Pinus radiata en el sur de Chile: ¿una herramienta para mitigar impactos ambientales negativos? Revista Chilena de Historia Natural 69: 393-400.

Oliet JA, DF Jacobs. 2007. Microclimatic conditions and plant morpho-physiological development within a tree shelter environment during establishment of Quercus ilex seedlings. Agricultural and Forest Meteorology 144: 58-72. DOI: https://doi.org/10.1016/j.agrformet.2007.01.012.

Oliet JA, R Blasco, P Valenzuela, MM de Blas, J Puértolas. 2019. Should we use meshes or solid tube shelters when planting in Mediterranean semiarid environments? New Forests 50: 267-282. DOI: https://doi.org/10.1007/s11056-018-9659-z.

Peri PL. 1998. Eficiencia de cortinas protectoras: Efectos de pa- rámetros estructurales en la reducción del viento, provincia de Santa Cruz, Argentina. Quebracho 6:19-26.

Piper F, G Baeza, A Zúñiga-Feest, A Fajardo. 2013. Soil nitrogen, and not phosphorus, promotes cluster-root formation in a South American Proteaceae, Embothrium coccineum. American Journal of Botany 100(12): 2328-2338. DOI: https:// doi.org/10.3732/ajb.1300163.

Premoli A. 2004. Variación en Nothofagus pumilio (Poepp. et Endl.) Krasser (Lenga). In Donoso C, A Premoli, L Gallo, R Ipinza eds. Variación Intraespecífica en las especies arbóreas de los bosques templados de Chile y Argentina. Santiago, Chile. Editorial Universitaria. p. 145-166.

Promis A, RB Allen. 2017. Tree seedlings respond to both light and soil nutrients in a Patagonian evergreen-deciduous forest. PLoS ONE 12(11): e0188686. DOI: https://doi. org/10.1371/journal.pone.0188686.

Promis A. 2018. Claros de dosel en bosques nativos y templados de Chile y Argentina; Conocimientos actuales y desafíos para el futuro. In Donoso PJ, A Promis, DP Soto eds. Silvicultura en Bosques Nativos. Experiencias en silvicultura y restauración en Chile, Argentina y el oeste de Estados Unidos. Estudios en Silvicultura de Bosques Nativos Volumen 2. Oregon, USA. OSU College of Forestry. p. 23-49.

Quintanilla V. 2005. Estado de recuperación del bosque nativo en una cuenca nordpatagónica de Chile, perturbada por grandes fuegos acaecidos 50 años atrás $\left(44^{\circ}-45^{\circ} \mathrm{S}\right)$. Revista de Geografia Norte Grande 34: 73-92. DOI: http://dx.doi. org/10.4067/S0718-34022008000100006.

Rands MR, WM Adams, L Bennun, SH Butchart, A Clements, D Coomes, A Entwistle, I Hodge, V Kapos, JPW Scharlemann, WJ Sutherland, B Vira. 2010. Biodiversity conservation: Challenges beyond 2010. Science 329(5997): 12981303. DOI: https://doi.org/10.1126/science.1189138.

Rovere AE, VR Chalcoff. 2010. Embothrium coccineum J. R. Forst. et G. Forst. Kurtziana 35(2): 23-33.

Saha S, C Kuehne, J Bauhus. 2016. Lessons learned from oak cluster planting trials in central Europe. Canadian Journal of Forest Research 47(2): 139-148. DOI: https://doi. org/10.1139/cjfr-2016-0265.

Shönenberger W. 2001. Cluster afforestation for creating diverse mountain forest structures - a review. Forest Ecology and Management 145(2): 121-128. DOI: https://doi. org/10.1016/S0378-1127(00)00579-X.

Schmidt H, G Cruz, A Promis, M Álvarez. 2003. Transformación de los bosques de lenga vírgenes e intervenidos a bosques manejados (Guía para los bosques demostrativos). Santiago, Chile. Facultad de Ciencias Forestales, Universidad de Chile. 60 p. (Publicaciones Misceláneas Forestales No 4).

Silva F. 2010. Flora agropecuaria de Aysén. Ministerio de Agricultura de Chile, Servicio Agrícola y Ganadero Región de Aysén. Coyhaique, Chile. Museo Nacional de Historia Natural. 520 p.

Till-Bottraud I, A Fajardo, D Rioux. 2012. Multi-stemmed trees of Nothofagus pumilio second-growth forest in Patagonia are formed by highly related individuals. Annals of Botany 110(4): 905-913. DOI: https://doi.org/10.1093/aob/mcs146.

Toro Manríquez M, A Promis, A Huertas Herrera, G Martínez Pastur. 2018. Influencia del micrositio y la exposición en la regeneración de bosques de Nothofagus pumilio afectados por Castor canadensis en Tierra del Fuego: un análisis exploratorio. Bosque 39(3): 431-440. DOI: https://doi. org/10.4067/S0717-92002018000300431. 
Toro Manríquez M, R Soler, MV Lencinas, A Promis. 2019. Canopy composition and site are indicative of mineral soil conditions in Patagonian mixed Nothofagus forests. Annals of Forest Science 76: 117. DOI: https://doi.org/10.1007/ s13595-019-0886-z.

Urretavizcaya MF, MJ Pastorino, V Mondino, LT Contardi. 2015. La Plantación con Árboles Nativos. In Chauchard L, MC Frugoni, C Nowak eds. Manual de Buenas Prácticas Forestales en el Noroeste de Patagonia. Buenos Aires, Argentina. p. 335-368.

Urretavizcaya MF, PL Peri, L Monelos, H Arriola, MF Oyharçabal, L Contardi, M Muñoz, R Sepúlveda, G Defosse. 2018. Soil and vegetation conditions in three burned Nothofagus pumilio forests in Argentina and experiences for their active restoration. Ecología Austral 28:383-399. DOI: https://doi. org/10.25260/EA.18.28.2.0.480.

Valenzuela P, E Arellano, J Burger, G Zegers, I Fernández. 2015. Bases para el desarrollo de un modelo de rehabilitación forestal en minería utilizando Nothofagus pumilio [Poepp.et Endl] Krasser. Anales Instituto Patagonia 43(1): 97-107. DOI: http://dx.doi.org/10.4067/S0718686X2015000100007.
Valenzuela PA, E Arellano, AJ Burger, P Becerra. 2016. Using facilitation microsites as a restoration tool for conversion of degraded grasslands to Nothofagus forests in Southern Patagonia. Ecological Engineering 95(1): 580-587. DOI: https://doi.org/10.1016/j.ecoleng.2016.06.116

Veblen TT, T Kitzberger, E Raffaele, DC Lorenz. 2003. Fire History and Vegetation Changes in Northern Patagonia, Argentina. In Veblen TT, WL Baker, G Montenegro, TW Swetnam eds. Fire and Climatic Change in Temperate Ecosystems of the Western Americas. Ecological Studies (Analysis and Synthesis), vol 160. New York, USA. Springer. p. 265-295.

Vidal OJ, M Aguayo, R Niculcar, N Bahamonde, S Radic, C San Martín, A Kusch, J Latorre, J Félez. 2015. Plantas invasoras en el Parque Nacional Torres del Paine (Magallanes, Chile): estado del arte, distribución post-fuego e implicancias en restauración ecológica. Anales Instituto Patagonia 43(1): 75-96. DOI: https://doi.org/10.4067/S0718686X2015000100006.

Whisenant SG. 1999. Repairing damaged wildlands: a processorientated, landscape-scale approach. Cambridge, UK. Cambridge University Press. 312 p. 
